LIVER

\title{
Carbamazepine accelerates liver regeneration in mice after acute injury
}

Carbamazepine-a commonly used anticonvulsant agent-has now been shown to stimulate hepatocellular proliferation and promote liver regeneration in mice after acute liver injury.

C57BL6/J mice were given an oral dose of carbamazepine or placebo before undergoing either a 70\% partial hepatectomy (PHx) or treatment with carbon tetrachloride (CCl4; a potent hepatotoxin). Within 2-5 days after PHx, the carbamazepine-treated mice had a greater liver:body weight ratio than mice given placebo. Moreover, carbamazepine led to an increased number of proliferative hepatocytes after PHx or CCl4 treatment.

The researchers went on to show that the hepatoproliferative effect of carbamazepine was dependent on the mTOR signalling pathway.
"Our most important finding is that carbamazepine accelerates the recovery of lost liver mass after partial hepatectomy, with enhancement of the number of proliferating hepatocytes," states Tsukasa Kawaguchi, first author of this study.

"We are now planning long-term carbamazepine administration in mouse models of chronic liver inflammation and fibrosis," Kawaguchi explains. "As carbamazepine has been administered safely in humans, further promising results from our studies will hopefully lead to a prospective clinical trial of this drug in patients," he adds.

Katherine Smith

Original article Kawaguchi, T. et al. Carbamazepine promotes liver regeneration and survival in mice. J. Hepatol. doi:10.1016/j.hep.2013.07.018 